\title{
Einstein-Podolsky-Rosen Constraints on Quantum Action at a Distance: The Sutherland Paradox
}

\author{
N. Cufaro-Petroni, ${ }^{1}$ C. Dewdney, ${ }^{2}$ P. R. Holland, ${ }^{2}$ \\ A. Kyprianidis, ${ }^{2}$ and J. P. Vigier $^{2}$ \\ Received May 29, 1986; revised January 21, 1987
}

\begin{abstract}
Assuming that future experiments confirm Aspect's discovery of nonlocal interactions between quantum pairs of correlated particles, we analyze the constraints imposed by the EPR reasoning on the said interactions. It is then shown that the nonlocal relativistic quantum potential approach plainly satisfies the Einstein causality criteria as well as the energy-momentum conservation in individual microprocesses. Furthermore, this approach bypasses a new causal paradox for timelike separated EPR measurements deduced by Sutherland in the frame of an approach by means of space-time zigzags with advanced potentials. It is finally demonstrated that this inherent quantum causal direct interaction establishes permanent EPR correlations which are always restricted to spacelike separations and are instantaneous only in the center-of-mass rest frame of the two-particle system.
\end{abstract}

\section{INTRODUCTION}

The flurry of experiments and theoretical papers devoted to the EPR paradox ${ }^{(1)}$ has reached a turning point with the experiment of Aspect et al., ${ }^{(2)}$ which violate Bell's inequalities and establish nonlocal correlations and/or interactions between the two measuring processes. If future experiments confirm this process and invalidate various criticisms, ${ }^{(3)}$ one will have to incorporate action at a distance in any interpretation of quantum mechanics and draw all possible epistemological and physical conclusions of this type of interaction.

\footnotetext{
${ }^{1}$ Istituto di Fisica, Università di Bari, Bari, Italy.

${ }^{2}$ Institut Henri Poincaré, Laboratoire de Physique Théorique, 11 rue P. et M. Curie, 75005 Paris, France.
} 
As one knows, anticipating these results, contemporary supporters of Bohr and Einstein's antagonistic positions have already proposed two specific opposed models of action at a distance to interpret EPR-type results and provide a nonlocal basis for quantum mechanics. The aim of the present paper is to summarize these conflicting models and confront their action-at-a-distance models with EPR's views on (1) energy momentum and angular momentum conservation in individual microprocesses and (2) causality and a new EPR paradox recently developed by Sutherland. ${ }^{(4)}$

\section{THE "RETROACTION-IN-TIME" MODELS}

Several physicists, in particular, Costa de Beauregard, ${ }^{(5,6)}$ have recently developed an approach to the EPR correlations based on the relativistic $S$-matrix scheme, which displays a full $C P T$ invariance. This approach is claimed to exhibit an "isomorphism between the formalism and its interpretative discourse," the latter being "the hallmark of a sound theory". ${ }^{(7)}$ In the following we will discuss the basic ingredients of this theory summarized in the following points:

(a) The theory should be completely Lorentz- and CPT-invariant. It should blindly read the formalism giving an absolute priority to it. Since only transition amplitudes exist, the concept of an evolving state vector is completely irrelevant. In fact, if $\left|\phi_{0}\right\rangle$ is the initial prepared state and $\left|\psi_{0}\right\rangle$ the measured state, then the system state $|S\rangle$ is neither in $\left|\phi_{i}\right\rangle=\left|U_{i 0} \phi_{0}\right\rangle$ (retarded evolution) nor in $\left|\psi_{i}\right\rangle=\left|U_{i 0}^{-1} \psi_{0}\right\rangle$ (advanced evolution) but only in a transiting state between the two, "these being the only states in which the system can be found". (7) Therefore "the evolving microsystem cannot be inside space-time-it is transiting beyond space-time (and beyond $K$-space). ${ }^{(7)}$

(b) The system is submitted to both the retarded action of preparation and the advanced action of measurement. This concept is $P T$ invariant and $C P T$-invariant and is a manifestation of the arrowless causality in the microlevel, a generalization of the Loschmidt $T$ symmetry of microcausality. ${ }^{(8)}$

(c) The macroscopic irreversibility is "factlike" but not "lawlike," i.e., not a strict consequence of the formalism.

(d) Physical causality should be merely identified with the conditional or transition probability concept $(A \mid C)$. The latter is derived from a conditional or transition amplitude $\langle A \mid C\rangle$ by the usual relation

$$
(A \mid C)=|\langle A \mid C\rangle|^{2}
$$


a basically timeless definition, exhibiting an intrinsic topological invariance with respect to time-zigzagging. ${ }^{(9)}$

(e) The formal solution of the Cauchy problem can be then written as

$$
\left\langle x^{\prime} \mid a\right\rangle=\left\langle x^{\prime} \mid x\right\rangle\langle x \mid a\rangle
$$

which can be interpreted as expanding the wave function upon the complete set of propagators with apexes on an arbitrary spacelike surface $\sigma$, the coefficients of the expansion being the values of the wave function on $\sigma .^{(7)}$

(f) The expression $\left\langle x^{\prime} \mid x\right\rangle$ represents the Jordan-Pauli propagator $D_{\mathrm{JP}}\left(x^{\prime}-x\right)=\left\langle x^{\prime} \mid x\right\rangle$, which is endowed with specific symmetries. In fact, if we denote by $D_{+}$the particle, $D_{-}$the antiparticle, $D_{\mathrm{R}}$ the retarded, and $D_{\mathrm{A}}$ the advanced propagator; then, on using the standard definitions, we obtain for the Jordan-Pauli propagator $D_{\mathrm{JP}} \equiv D_{+}-D_{-}=D_{\mathrm{R}}-D_{\mathrm{A}}$ and for the Feynman propagator $D_{\mathrm{F}} \equiv D_{\mathrm{R}}+D_{-}=D_{\mathrm{A}}+D_{+}$, which satisfy $\square D_{\mathrm{JP}}=0$ and $\square D_{\mathrm{F}}=\delta\left(x^{\prime}-x\right)$. We can readily deduce the invariance of $D_{\mathrm{JP}}$ under $P=-T=C=1$ and of $D_{\mathrm{F}}$ under $P=C T=1 .{ }^{(7)}$

(g) Finally, the $D_{\mathrm{F}}$ automatically entails the exponential decay of higher energy levels in a predictive calculation and an exponential build-up of the higher energy levels in a blind retrodictive calculation. "Therefore (and contrary to a more or less existing feeling) there is no causality asymmetry whatsoever built in to the Feynman propagator". ${ }^{(7)}$

We proceed with the presentation of the reasoning and the consequent interpretation of the quantum results. A far-reaching implication of the preceding line of thought is obvious and concerns the EPR situations and the paradoxical character of quantum correlations: In EPR correlations adjustable parameters exist at the measurement positions and not in the position of common preparation of the system. The link of the correlation is the Feynman zigzag consisting of two timelike vectors with a relay at the source in the past ${ }^{(10)}$ because no other physical link exists between them. ${ }^{(11)}$ This link is furthermore insensitive to the spacelike or timelike character of the separation of the two measuring processes. ${ }^{(12)}$ Consequently, a zigzagging mechanism is the only possible explanatory pattern for the EPR correlations.

In what follows we do not intend to argue step by step every point discussed in this brief résumé. What we wish to present instead are some crucial arguments showing the fallacies, unauthorized extrapolations, and wrong assertions of this approach. 


\section{GENERAL OBJECTIONS AND CRITICISM}

First of all, the quantal evolution is in fact a unitary evolution from the original preparation. The Copenhagen interpretation introduces the collapse concept to account for measurement but there is no real need for that, since Bohm ${ }^{(14)}$ and $\mathrm{Cini}^{(15)}$ have deduced the "factlike" irreversibility by means of a realistic interaction process with the measuring device. Measurement as a spectral decomposition procedure of the wave packet and the particle entering one of the resulting subpackets accounts perfectly well for the observed phenomena. It proves the inconsistency of the collapse concept and excludes the even more inconsistent "collapse + retrocollapse" mechanism, which in any case does not result from the formalism but is simply added by hand.

Secondly, the CPT invariance of the formalism simply denotes the existence of antiparticles as a constituent element of the theory in the relativistic frame, a fact that enables an alternative mathematical approach ( $E<0$ propagating along $t<0$ ), but which definitely does not allow a juggling with symmetries and propagators at free will. Pauli-Jordan propagators do not reproduce the physical context since they propagate positive energies backward in time. This propagator is separately $C, P, T$ invariant, satisfying the requirements of Costa de Beauregard. But the Feynman propagator needed for the $S$-matrix formulation is only $P$ - or $C T$-invariant, connecting thus time reversal with charge conjugation, or the particle to antiparticle transition, which clearly contradicts the $P T$ invariance required under (b) for the combined advanced and retarded action on a system. Clearly, one can easily conceive the action of antiparticle contributions coming from the future in a set of Feynman graphs for a given process. We only have to use the conjugated physical picture in order to see that "apparent" influences from the future can be simply restated in the following form: An event at $x^{\mu}$ is influenced by the inflow of particle contributions from the past and the outflow of particles with opposite charge and opposite space-momenta into the future (instead of inflow of antiparticles from the future), which is equivalent to saying that creation/annihilation processes in a position $x^{\mu}$ do influence the events at $x^{\mu}$. No need exists for retrocausation or whatsoever, because no retrocausation is implied by the use of the Feynman propagator. Furthermore, this fact cannot be circumvented by an escape toward the anti-Feynman propagator using the opposite convention in the assignment of the particle or antiparticle status. Mathematical conventions do not change physical reality unless we do not use them consistently. And still there is a basic difference between a retrodictive calculation and a retroactive propagation, the former concerning knowledge and the latter a physical 
occurrence. The Feynman propagator does imply an irreversible entropy increase in future particle evolution, and no particle evolution toward the past is allowed. Causality has a positive time arrow, and this fact does not change by using an antiparticle (mathematical) picture. Temporal asymmetry is inscribed in the Feynman propagator formalism in whatever (particle/antiparticle) convention, the only escape from this fact being the use of a time-symmetric propagator, i.e., the Jordan-Pauli type, which is in fact introduced by Costa de Beauregard in the solution of the Cauchy problem [cf. point (e) above]. This is of course not equivalent to the standard approach and has been criticized elsewhere. ${ }^{(16)}$

Thirdly, using explicitly a $T$-irreversible or $P T$-reversible approach, one can construct a model for EPR correlations, by shooting positive energies backward in teme. This model of "antitelegraph mechanism"(13) produces the desired correlations, but at what expense? Conservation laws are violated at the source, since positive energy appears from the future with no apparent causes. This model is not rendered more credible by some kind of circular argument of the kind that "causes exist only at the measuring positions and there is no other physical link than the zigzag in time", (11) where the proof of the hypothesis is based on the hypothesis itself. Furthermore, this zigzag should not be labeled as a Feynman zigzag, since the latter are particle-antiparticle processes and no such mechanism is evidently advanced by the "retroaction" approach. Recami ${ }^{(17)}$ has indeed shown that particle-antiparticle exchange can be denoted by $P T$ and $C$ reversal, deducing from this that causality is strictly retarded, contrary to the assumptions of Costa de Beauregard. In fact, let us simply mention the following as an additional argument in this context:

(1) The $T$-invariance-breaking interactions such as $K^{\circ} \rightarrow 3 \Pi$ decays, which are not reproduced by the use of the $D_{\mathrm{JP}}$;

(2) The spontaneous decays of the vacuum into two systems propagating forward in time, one with positive and the other with negative energy, which is evidently implied by the use of $D_{\mathrm{JP}}{ }^{(16)}$;

(3) Finally, that retarded causality implies dispersion relations which have been derived and checked experimentally. ${ }^{(18)}$

These points and the preceding arguments due to a consistent (not "blind") reading of the formalism lead us indeed to discard Costa de Beauregard's "extended relativistic micro-invariance", ${ }^{(19)}$ his trespassing the "taboos" against faster-than-light or backward-in-time telegraphing, and his combination of active + retroactive (acausal) determinism. 


\section{A SPECIFIC OBJECTION: THE SUTHERLAND PARADOX}

But a very simple and elegant refutation of this approach is due to one of the former proponents of "retroaction model": Sutherland ${ }^{(4)}$ produces a "paradox" for a measurement on EPR-correlated pairs depending on their respective timelike or spacelike separation. The paradox holds equivalently for the quantum calculus, as well, unless additional restrictions are introduced. Let us summarize the Sutherland paradox as follows: Figure 1 shows the space-time picture of a decay at $D$ of a spin-zero state to two spin-one-half particles. Spin measurements are performed at $M_{1}$ and $M_{2}$ or $M_{1}^{\prime}$ and $M_{2}$, the differences lying respectively in the spacelike or timelike character of the intervals. According to the quantum formalism, the results on $M_{1}$ and $M_{2}$ as well as those on $M_{1}^{\prime}$ and $M_{2}$ are always correlated since there are no different predictions depending on the spacelike or timelike character of the separating interval. Then, for a timelike separation $M_{1}^{\prime} M_{2}$, the following reasoning can be advanced:

(a) The result at $M_{2}$ depends on the choice at $M_{1}^{\prime}$ : Therefore, for some pairs of particles, it is true that choosing a direction $\omega_{1}$ for the $M_{1}^{\prime}$ measurement would yield $+\frac{1}{2} h$ at $M_{2}$, and choosing $\omega_{2}$ at $M_{1}^{\prime}$ would yield $-\frac{1}{2} \hbar$ at $M_{2}$.

(b) Since $M_{1}^{\prime} M_{2}$ is timelike, we can in principle signal from $M_{2}$ to $M_{1}^{\prime}$, instructing the measuring device at $M_{1}^{\prime}$ to choose a direction at will.

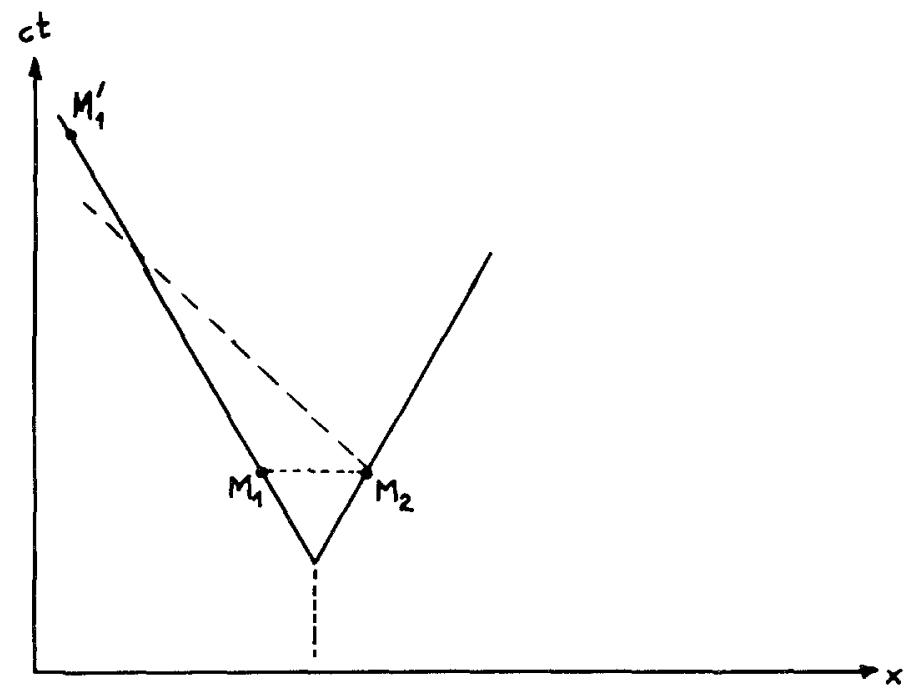

Fig. 1. Space-time picture of a decay $D$ : Spin measurements are performed at $M_{1}$ and $M_{2}$ or $M_{1}^{\prime}$ and $M_{2}$, the difference lying, respectively, in the spacelike or timelike character of the intervals. 
(c) Then, when $+\frac{1}{2} \hbar$ is observed at $M_{2}$, we instruct $M_{1}^{\prime}$ to choose $\omega_{2}$, and, for $-\frac{1}{2} h$ at $M_{2}$, we instruct $M_{1}^{\prime}$ to choose $\omega_{1}$. Using point (a) above, we conclude that neither $+\frac{1}{2} h$ nor $-\frac{1}{2} \hbar$ is a consistent outcome for the particle $M_{2}$, the paradox arising unpredictably after trying various pairs of directions.

This paradox indeed raises a serious difficulty in Costa de Beauregard's approach, as we can be convinced by looking at his comments thereon:

“... an EPR correlation amplitude is a conditional amplitude valid if and only if the two measurements at $A$ and $C\left(M_{1}^{\prime}\right.$ and $M_{2}$ in our notation), as expressed in the formula, are actually performed. What is relevant for the interpretation is what is written down in the correlation formula, nothing else. How and when the decision concerning the setting of the instrument is made is not in the formulas, and therefore is not relevant." ${ }^{(13)}$

Sutherland's arguments, however, show that it is in fact relevant, since certain configurations of performed measurements do produce paradoxical (i.e., unacceptable) results. Therefore, this kind of global statement can be hardly considered as an adequate reply to the criticism. Even if it implies that predicted results are obtained after the setting of the instruments, the paradox still persists because no regulation forbids the final setting of the second device at $M_{1}^{\prime}$ by means of a signal after the result at $M_{2}$ has been obtained. Or does this approach suggest that, by some kind of retroaction, no definite result will be visible at $M_{2}$ if a signal is going to be sent to $M_{1}^{\prime}$ after the spin measurement on particle 2 ?

\section{A SOLUTION OF THE PARADOX: THE CAUSAL QUANTUM POTENTIAL MODEL}

In fact the solution of this paradox can be achieved along the lines of a restriction advanced tentatively by Sutherland himself $f^{(4)}: M_{1}$ must affect $M_{2}$ when $M_{1}$ is outside the forward light cone of $M_{2}$ but not when it is inside. This is exactly the constraint obeyed by the nonlocal quantumpotential model which we will briefly discuss in what follows.

First of all this model satisfies Einstein's causality constraints:

(1) Invariance under the causality $\operatorname{group}^{(20)} G=T \otimes \mathscr{L} \uparrow \otimes D+P$, where $T=$ translations, $\mathscr{L} \uparrow=$ orthochronous Lorentz transforms, $D=$ dilatations, and $P=$ space symmetries.

(2) Only positive energy propagates forward in time.

(3) All subsequent motions can be derived from a set of initial conditions on a spacelike surface, i.e., the Cauchy problem can be solved unequivocally. 
Furthermore it obeys two additional "EPR constraints":

(a) Energy-momentum and angular momentum are absolutely conserved in each individual microprocess.

(b) If one can predict with certainty (probability 1) a given result without in any way disturbing the system, then an objective element of physical reality corresponds to this prediction.

Following Bohm ${ }^{(21)}$ and Vigier, ${ }^{(22)}$ this model is based on the Madelung-type decomposition of the relativistic quantum equations. For the sake of clarity, we will briefly discuss the rough lines for the case of the spin-zero Klein-Gordon equation, indicating the important deviations that appear when we are dealing with relativistic equations of spinning particles (Feynman-Gell-Mann equation for spin $\frac{1}{2}$, ${ }^{(23)}$ Proca equation for spin $\left.1^{(24)}\right)$. The Klein-Gordon equation $\left(\square+m^{2}\right) \psi\left(x^{\mu}\right)=0$ is decomposed by means of the Ansatz $\psi=e^{P+i \frac{S}{h}}$ in to a set of two equations (real and imaginary part, respectively), namely a Hamilton-Jacobi type equation,

$$
\frac{m^{2} c^{2}}{\hbar^{2}}-\frac{1}{\hbar^{2}} \partial_{\mu} S \partial^{\mu} S+\left(\square P+\partial_{\mu} P \partial^{\mu} P\right)=0
$$

where $Q=\left(\square P+\partial_{\mu} P \partial^{\mu} P\right)$ is the relativistic expression for the quantum potential of Bohm, ${ }^{(21)}$ and a continuity equation

$$
\partial_{\mu}\left(e^{2 P} \frac{\partial^{\mu} S}{m}\right)=0
$$

In this model, particles have timelike trajectories with $v^{\mu}=\partial^{\mu} S / m$, this property being obtained by a physical restriction on the ensemble of mathematical solutions of the Klein-Gordon equation. ${ }^{(25)}$ The trajectories obtained hereby are determined by the context-dependent quantum potential, which ensures the existence of the known quantum statistical distributions, ${ }^{(26)}$ and are always associated with positive probabilities and positive energies moving forward in time, ${ }^{(27)}$ thus satisfying Einstein's causality conditions (1) and (2). Finally, in the case of $N$-particle systems, the existence of nonlocal correlations, described by the nonlocal quantum potential, has been shown to conform with a causal solution of the Cauchy problem according to Einstein's third requirement. ${ }^{(28)}$ The conservation laws are re-established in individual microprocesses where in general the quantum potential (or the spindependent quantum potential and "quantum torques," i.e., a spin-spin action at a distance in the case of spinning particles) has to be explicitly included in the corresponding considerations. Finally, a physical reality according to EPR constraint (2) has to be 
ascribed to physical observables such as momentum, spin, etc. independent of any measurement process.

In the present case it is sufficient to examine the two-particle system in the frame of this model since it corresponds to the given experimental situation. In fact, we consider a composite wave field $\psi\left(x_{1}, x_{2}\right)$ in configuration space satisfying the free-particle wave equations $(c=\hbar=1)$

$$
\left(\square_{i}+m_{i}^{2}\right) \psi\left(x_{1}, x_{2}\right)=0, \quad i=1,2
$$

Writing $\psi=\exp \left[P\left(x_{1}, x_{2}\right)+i S\left(x_{1}, x_{2}\right)\right]$ along with $P_{i}^{\mu}=\partial_{i}^{\mu} S(i=1,2)$, we deduce from the real part of Eq. (3) that the system of two Klein-Gordon relativistic free particles can be mapped onto a system of two relativistic free particles submitted to nonlocal potentials ${ }^{(29)}$

$$
H_{i}=\frac{P_{i}^{2}}{2}+Q=\frac{m_{i}^{2}}{2}, \quad i=1,2
$$

where $Q$ is the map of the quantum potential in relativistic particle dynamics and is independent of the values of $m_{1}$ and $m_{2}$. The difference of the two equations (4) yields

$$
\left(P_{1 \mu}+P_{2 \mu}\right)\left(P_{1}^{\mu}-P_{2}^{\mu}\right)=m_{1}^{2}-m_{2}^{2}
$$

Furthermore, we have $P_{1 \mu}+P_{2 \mu}=P_{\mu}$, where $P_{\mu}$ is the four-momentum of the center of mass $(\mathrm{CM})$. A transformation to the $\mathrm{CM}$ rest frame $\left(P^{0}, \underline{P}=0\right)$ where $P_{1}^{k}=-P_{2}^{k}$ and $P_{1}^{0}+P_{2}^{0}=P^{0}$ casts Eq. (5) into the form $P_{1}^{0}-P_{2}^{0}=\left(m_{1}^{2}-m_{2}^{2}\right) / P^{0}$ and yields

$$
P_{1}^{0}=\frac{P^{0}}{2}+\frac{m_{1}^{2}-m_{2}^{2}}{2 P^{0}}, \quad P_{2}^{0}=\frac{P^{0}}{2}-\frac{m_{1}^{2}-m_{2}^{2}}{2 P^{0}}
$$

Obviously in this frame the energies of the particles are constants of the motion.

Finally, it has been shown ${ }^{(29)}$ that, in order to map the free Klein-Gordon system on a system of relativistic particles tied by a nonlocal potential, the latter has to exhibit the following functional dependence (with $z^{\mu}=x_{1}^{\mu}-x_{2}^{\mu}$ ):

$$
Q(\tilde{z})=Q\left(z^{\mu}-\frac{\left(z_{v} q^{v}\right) q^{\mu}}{P^{2}}\right)
$$

A transformation to the CM rest frame yields $\tilde{z}_{C M}^{\mu}=\left(0, z^{k}\right)$ and $Q(\tilde{z})=Q\left(z^{k}\right)$. The quantum potential $Q$ does not depend on the relative time and its action is instantaneous with no exchange of energy between 
the two particles in this frame according to Eq. (6). Using the functional dependence of the quantum potential on the projection of the space-time separation of particles 1 and 2 , namely $\tilde{z}$ and the obvious property $P^{\mu} \tilde{z}_{\mu}=0$, we can now convince ourselves that the action-at-a-distance nonlocal correlation of particles 1 and 2 exists only on a spacelike surface perpendicular to the $\mathrm{CM}$ four-momentum $P^{\mu}$ which is a timelike vector. It becomes instantaneous only in the $\mathrm{CM}$ rest frame where $\tilde{z}^{\mu}=\left(0, z^{k}\right)$, which is due to the fact that $P^{\mu}=\partial S / \partial X_{\mu}$ with $X^{\mu}=\left(m_{1} x_{1}^{\mu}+m_{2} x_{2}^{\mu}\right) /$ $\left(m_{1}+m_{2}\right)$, i.e., the $\mathrm{CM}$ coordinate, coincides with the surface of constant phase with respect to $X$; i.e., $S(X, \tilde{z})=S(\tilde{z})$.

For the sake of completeness, we wish to mention that such an action at a distance, manifest in a relativistic dynamics version of the quantum equation, ${ }^{(30)}$ is perfectly compatible with Einstein's causality, as a consequence of the fact that the particle Hamiltonians obey a constraining predictivity condition $\left\{H_{1}, H_{2}\right\}=0$, which ensures the existence and timelikeness of the particle world lines. This property has been recently extended to the quantum $N$-body system. ${ }^{(31)}$ Of course, this simple and intuitively transparent presentation does not account for the spincorrelation properties, but it can be easily shown that the qualitative result of this section, namely action at a distance for spacelike separation, remains valid also for the case of spinning particles. ${ }^{(23,24)}$

We are now in a position to solve the Sutherland paradox in the frame of the quantum potential model of quantum mechanics. This solution rests on the following points:

(1) In the "retroaction approach" it is usually claimed that composite states are nonseparable and unanalyzable and reality is created if a measurement process is really performed. Contrary to that, our model rests on an incommensurable physical assumption: The system + context (experimental arrangement, measuring devices, etc.) build a whole due to permanently active nonlocal action-at-a-distance correlations between existing real physical (observable) variables of its constituent elements, independent of any attempted or performed measurement process. Nevertheless, the system is analyzable, at least in principle. To see things more clearly, let us examine the given paradox in our scheme. The latter implies: (a) The existence of spin values for each particle independently of any measurement. For an explicit calculation in the relativistic frame, we refer to Refs. 22 and 23, and, for a nonrelativistic approach by means of a causal interpretation of the Pauli equation, similar calculations for different experimental arrangements can be found in Refs. 32 and 33. (b) The spin vectors of the two particles in a singlet state are correlated by a spin-spin action at a distance, or a "quantum torque," so that they always remain in 
an opposite direction. This point seems to be confirmed by a calculation of the total angular momentum of a system consisting of two particles in a singlet state and of a measuring device operating on the spin of one of the particles. $^{(34)}$ (An alternative approach consists in dropping the usual idealized measurement theory, as discussed, for example, in Refs. 35 and 36.) This calculation yields a nonconservation of the total angular momentum. Supposing that an interaction of a particle spin with a measuring device (e.g., Stern-Gerlach apparatus) is perfectly local and consists in a twisting process of the spin due to the classical interaction with the Stern-Gerlach field and the "quantum torque," we deduce that the "quantum torque" twists the second particle's spin to a corresponding direction so that the singlet state is preserved. The apparent violation of angular-momentum conservation is a consequence of the fact that in the attempted balance the "quantum torque" has been neglected; conservation is re-established if it is explicitly taken into account.

(2) The quantum measurement results are assumed to correspond to real interactions between the apparatus and the measured particles. No wave packet collapse exists in this model. Since the two particles are permanently nonlocally spin-correlated (singlet state) in spacelike separations, and if spacelike separated measurements are performed on particles 1 and 2 , then, due to the existence of a "quantum torque," the measurement results $M_{1}$ and $M_{2}$ must possess this correlated feature depending on the choice of polarization directions. The situation is quite different if the two measurements are separated by a timelike interval, $M_{2}$ preceding $M_{1}^{\prime}$ in the time ordering. Then at $M_{2}$, due to the interaction with the device, the spin $S_{2}$ rotates and acquires a certain value of the $z$ component, say $+\frac{1}{2} h$, and consequently the "quantum torque" twists $S_{1}$ which is spacelike separated from $S_{2}$ into a corresponding value for $S_{z_{1}}=-\frac{1}{2} \hbar$, which is not measured. The measurement on $M_{2}$ is an objective procedure, say a Stern-Gerlach splitting of the original wave packet in two packets, where only the spin-up packet is occupied by particle 2 . This measurement result rest on the action of the "quantum torque," which depends on the system as a whole (i.e., particles 1 and 2 and apparatus). ${ }^{(32)}$ The system remains on the correlated singlet state, i.e., particles 1 and 2 interact nonlocally, after particle 2 has emerged from the measuring device. The subsequent measurement at $M_{1}^{\prime}$ on particle 1 can be conceived along the same scheme, and the results depend again on the context of the experiment and the "quantum torque" between the two particles. In any case the spin values $S_{1}$ and $S_{2}$ remain singlet-correlated. However, this is not the point envisaged by the "paradox," because the latter is concerned with the correlation of the measurement results $M_{1}^{\prime}$ and $M_{2}$. Concerning this point, the following can 
be stated: If, for example, the relaxation time of the $2(\uparrow)-1(\downarrow)$ state is big with respect to the time separation of the events in the observer frame, then the separation of the spin-up and spin-down packets after passage through $M_{2}$ persists and a subsequent $M_{1}^{\prime}$ measurement will yield a correlated result with respect to the $M_{2}$ measurement. No direct correlation exists between the measuring devices, but the correlation of results is mediated via the fixing of the $S_{2}$ value after the passage through $M_{2}$. On the contrary, if the relaxation time is very small with respect to the time separation of the two measurements, or if the splitting wave packets with spin $\uparrow$ and $\downarrow$ of particle 2 recombine, then the subsequent $M_{1}^{\prime}$ measurement will be decorrelated from the $M_{2}$ measurement, due to the loss of the splitting characteristics after $M_{2}$, although at $M_{1}^{\prime}, S_{1}$ and $S_{2}$ are still singlet-correlated. Let us stress once more that since action at a distance is limited to space like directions, any nonlocal influence exists between spacelike separated particles and not between timelike separated measurements. It is really incomprehensible to think of measurement results as being influenced by the preexisting settings of a measuring device supposed to operate in the future light cone of an actually occurring measurement on one of the particles. The Sutherland paradox is probably tied to the quantum measurement theory and its shortcomings; it is not real but only conceptual. It is tied to the basic philosophical assertion that reality is created by measurement, which lies also at the origin of the completely unfounded and, in our opinion unjustified, extrapolations, of the time-retroactive type. By contrast, our model meets the requirement imposed by Sutherland, namely a different behavior for timelike and spacelike separations between the measurement processes, because it is not the measurement processes which are nonlocally connected but the particles themselves. Even more so, it is plausible because it does not rest on an a priori assumption but deduces the results out of a nonlocal causal action-at-a-distance mechanism. Finally it does not contradict "quantum mechanics," as incorrectly implied by Sutherland, ${ }^{(4)}$ but only certain assertions of a highly controversial topic such as is the "quantum measurement theory," a common label for a set of mostly controversial and, in any case, incoherent calculatory recipes with one common denominator: the "reality creation by measurement" assumptions.

\section{CONCLUSIONS}

The fate of the EPR paper is indeed quite astonishing, since it is now at the very center of an important epistemological confrontation on the physical and philosophical implication of quantum mechanics. Its discussion, originally limited to the question of the complete (or incomplete) 
character (Bohr believed in completness, EPR did not) of the quantum mechanical description, has blossomed in successive steps inte

- the question of the local (or nonlocal) character of quantum mechanical correlations (Bohr believed in nonlocality, Einstein and de Broglie did not);

- the question of the existence (or not) of local (or nonlocal) hidden variables subtending quantum mechanical statistics. (Einstein and de Broglie believed in hidden variables, Bohr did not);

- the question of whether particles move (or not) in real space-time (Einstein and de Broglie believed in space-time motions, Bohr did not).

The discussion was then (later) enlarged to questions

- on the nature of the wave particle dualism (are micro-object waves or particles as claimed by Bohr and Heisenberg or waves and particles simultaneously as proposed by Einstein and de Broglie);

- on the question of the collapse (or not) of waves in measurement process;

- on the existence (or not) of micro-objects independently of conscious observations (Einstein and de Broglie belived in this existence, Bohr did not);

- on the existence (or not) of space-time itself also independently of conscious observations;

- on the causal (noncausal) nature of the physical laws themselves.

This is a remarkable fate indeed. All the more remarkable is that, on all successive questions raised, Bohr and Einstein (as can be readily checked by the reader) have expressed absolutely antagonistic positions.

The new fact is that technology has now evolved to a stage where these formerly purely theoretical discussions (with accompanying Gedanken experiments) now can be tested by engineer-realizable experiments, i.e., can be solved (as they should) by experiment. For example

- a final check of the nonlocal correlations in Aspect EPR-type experiments will definitely answer the raised objections;

- the question whether or not particles move in real space-time and the validity of wave-particle dualism will be probably answered by timedependent neutron interferometry experiments. ${ }^{(37)}$

The fate of the Bohr-Einstein debate thus lies in the hands of the experimental physicists. 


\section{ACKNOWLEDGMENTS}

The authors are very grateful to Dr. O. Costa de Beauregard for advanced communication of his opinion on the Sutherland paradox. They also want to thank the French Government and Italian INFN for grants which made this research possible.

\section{REFERENCES}

1. A. Einstein, B. Podolsky, and N. Rosen, Phys. Rev. 47, 777 (1935).

2. A. Aspect, P. Grangier, and R. Roger, Phys. Rev. Lett. 47, 460 (1981); A Aspect, J. Dalibard, and R. Roger, Phys. Rev. Lett. 49, 1804 (1982).

3. F. Selleri, Phys. Lett. A 108, 197 (1985); T. W. Marshall, E. Santos, and F. Selleri, Phys. Lett 98, 5 (1983); A. Garuccio and F. Selleri, Phys. Lett. A 103, 99 (1984).

4. R. I. Sutherland, Nuovo Cimento B 88, 114 (1985).

5. O. Costa de Beauregard, Compt. Rend. Acad. Sci. (Paris) 236, 1632 (1953); Nuovo Cimento B 42, 41 (1977); Nuovo Cimento B 51, 267 (1979).

6. H. Stapp, Nuovo Cimento B 29, 270 (1975); W. C. Davidon, Nuovo Cimento B 36, 34 (1976); J. Rayski, Found. Phys. 9, 217 (1979); J. G. Cramer, Phys. Rev. D. 22, 362 (1980); C. W. Rietdijk, Found. Phys. 8, 615 (1978); 11, 783 (1981); R. I. Sutherland, Int. I. Theor. Phys. 22, 377 (1983).

7. O. Costa de Beauregard, in Foundations of Quantum Mechanics in the Light of New Technology, S. Kamefuchi et al., eds. (Physical Society of Japan, Tokyo, 1983).

8. O. Costa de Beauregard, Ann. Fond. L. de Broglie 6, 329 (1981).

9. O. Costa de Beauregard, "Causality as identified with conditional probability and quantal nonseparability," in Microphysical Reality and Quantum Formalism, F. Selleri, G. Tarozzi, and A. van der Merwe, eds. (Reidel, Dordrecht, 1987).

10. O. Costa de Beauregard, Found. Phys. 15, 871 (1985).

11. O. Costa de Beauregard, Nuovo Cimento Lett. 29, 551 (1980).

12. O. Costa de Beauregard, Nuovo Cimento Lett. 25, 91 (1979).

13. O. Costa de Beauregard, "Comments on a recent article by R. I. Sutherland," IHP preprint, December 1985.

14. D. Bohm and B. J. Hiley, Found Phys. 14, 255 (1984).

15. M. Cini, Nuovo Cimento B 73, 27 (1983).

16. F. Selleri and J. P. Vigier, Nuovo Cimento Lett. 29, 7 (1980).

17. E. Recami, "Classical Tachyons and Possible Applications: A Review," Ist. Naz. Fis. Nuc./AE-84/8, 1984.

18. G. Källen, Elementary Particle Physics (Addison-Wesley, Reading, Massachusetts, 1969).

19. G. Lüders, Z. Phys. 133, 325 (1952).

20. E. C. Zeemann, J. Math. Phys. 5, 490 (1964).

21. D. Bohm, Phys. Rev. 85, 168, 180 (1952).

22. J. P. Vigier, Astr. Nachr. 303, 55 (1982).

23. N. Cufaro-Petroni, Ph. Gueret, A. Kyprianidis, and J. P. Vigier, Phys. Rev. D 31, 3157 (1985) and references quoted therein.

24. N. Cufaro-Petroni and J. P. Vigier, Phys. Lett. A 73, 289 (1979).

25. A. Kyprianidis, Phys. Lett. A. 111, 111 (1985).

26. A. Kyprianidis, D. Sardelis, and J. P. Vigier, Phys. Lett. A 100, 228 (1984); N. CufaroPetroni, A. Kyprianidis, Z. Maric, D. Sardelis, and J. P. Vigier, Phys. Lett. A 101, 4 (1984). 
27. N. Cufaro-Petroni, C. Dewdney, P. R. Holland, A. Kyprianidis, and J. P. Vigier, Phys. Lett. A 106, 368 (1984); P. R. Holland, A. Kyprianidis, and J. P. Vigier, Phys. Lett. A 107, 376 (1984). Ph. Gueret, P. R. Holland. A. Kyprianidis, and J. P. Vigier, Phys. Lett. A 107, 379 (1985).

28. N. Cufaro-Petroni, C. Dewdney, P. R. Holland, A. Kyprianidis, and J. P. Vigier, Lett. Nuovo Cimento 42, 285 (1985).

29. N. Cufaro-Petroni, Ph. Droz-Vincent, and J. P. Vigier, Lett. Nuovo Cimento 31, 415 (1981).

30. Ph. Droz-Vincent, Phys. Rev. D 19, 702 (1979) and references quoted therein.

31. A. Garuccio, A. Kyprianidis, and J. P. Vigier, Nuovo Cimento B 83, 135 (1984).

32. C. Dewdney, P. R. Holland, A. Kyprianidis, and J. P. Vigier, "Trajectories and spin vector orientations in the causal interpretation of the Pauli equation," IHP preprint, December 1985.

33. C. Dewdney, P. R. Holland, A. Kyprianidis, and J. P. Vigier, "Spin superposition in neutron interferometry," IHP preprint, December 1985.

34. N. Cufaro-Petroni, A. Garuccio, F. Selleri, and J. P. Vigier, C. R. Acad. Sci. (Paris), 290, $111(1980)$.

35. E. P. Wigner, Z. Phys. 133, 101 (1952).

36. H. Araki and M. Yanase, Phys. Rev. 96, 208 (1954).

37. J. Summhammer, G. Badurek, and U. Kischko, Phys. Lett. A 90, 110 (1982); G. Badurek, H. Rauch, J. Summhammer, U. Kischko, and A. Zeilinger, J. Phys. A 16, 1133 (1983); J. Summhammer, G. Badurek, H. Rauch, U. Kischkho, and A. Zeilinger, Phys. Rev. A 27, 2523 (1983); G. Badurek, H. Rauch, and J. Summhammer, Phys. Rev. Lett. 51, 1015 (1983). 